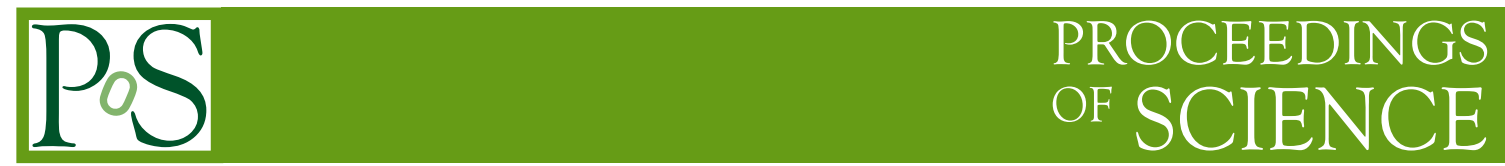

\title{
Prospects for neutrino oscillation parameters
}

\section{Patrick Huber*}

Center for Neutrino Physics, Virginia Tech, Blacksburg, USA

E-mail: pahuber@vt.edu

In this contribution we discuss the future of the global long-baseline neutrino oscillation program. The case is made that our current lack of understanding of neutrino-nucleus interactions is a serious challenge which will need to be met with new experimental initiatives in neutrino scattering.

Neutrino Oscillation Workshop

4 - 11 September, 2016

Otranto (Lecce, Italy)

\footnotetext{
* Speaker.
} 


\section{Introduction}

The discovery of neutrino oscillation, awarded the 2015 Nobel prize in physics, is one of the great discoveries of our time. Apart from finding that neutrinos have mass, we also have been blessed with large mixing angles in both solar and atmospheric neutrinos as well as a large value of $\theta_{13}$. All of which are necessary ingredients to allow for the search of $\mathrm{CP}$ violation in neutrino oscillations, since the absolute size of CP effects is suppressed by the smallest of the mixing angles and by the ratio of two mass squared differences. The study of $\mathrm{CP}$ violation in turn requires the ability to perform an appearance measurement, and given our lack of proper technology to efficiently create or detect $v_{\tau}$, this implies the use of $v_{e}$ and $v_{\mu}$. The need to involve $v_{\mu}$ in turn requires to use energies in the 100 's of $\mathrm{MeV}$ and above and this in turn results in long baselines of 100 's $\mathrm{km}$ and more.

The event rate for $v_{e} \rightarrow v_{\mu}$ or $v_{\mu} \rightarrow v_{e}$ appearance in leading order is proportional to $\sin ^{2} 2 \theta_{13}$ and thus, the fact that $\theta_{13}$ is large is good news: it allows to obtain a sufficiently large event sample using conventional pion-decay, horn-focused neutrino beams, which we have been using for more than 3 decades. It still requires, however, to push this technology to its limits by using MW-level proton beams and very large detectors of at least the size of Super-Kamiokande. These long-baseline experiment are characterized in terms of megawatts, kilotons and decades. This is a new scale of effort in neutrino physics and makes neutrino physics, in its sheer experimental scope and scale, much more similar to traditional accelerator based science, like for instance the LHC program.

Indeed, DUNE is expected to start data taking roughly a decade from now and to run for a decade and to absorb a very large fraction of the resources of its host country and the international neutrino community. Also for many scientists this will be the only major experiment during their career. The sheer scale of these new neutrino experiments also implies that failure to make a major discovery is not an option. For small-scale neutrino experiments like the ones in the Booster neutrino beam at Fermilab or reactor neutrino experiments not finding anything or being preempted by some other measurement is a completely acceptable outcome because there are many experiments at this scale. As long as some of these experiments make discoveries or provide measurements, science and importantly, along with it, the careers of those conducting these experiments can progress. The bargain each scientists strikes with a generational experiment like the LHC is based on the conviction that the science goal is worthy of a lifetime of struggle and the realization that this is the only way to achieve this particular science goal. Implied in this bargain is the understanding that both, the worthiness of the science goal and the unique ability to achieve it by this one specific experiment, will endure over the decades it takes to carry out this program.

For the LHC the science goal was the discovery of the Higgs boson and to find New Physics, either in the form of new degrees of freedom or by some other breakdown of the Standard Model. It also is understood that this would require particle collisions at unprecedented energies and after the global community had settled on the LHC is also was clear that this will be the only machine in this energy regime for the foreseeable future. The Higgs has been discovered and the search for New Physics is ongoing, with a major upgrade of the machine underway - a roundabout success.

For long-baseline oscillation experiments the science goal is the discovery of leptonic CP violation and the search for New Physics by precisely testing the three-flavor oscillation framework. 
However, this is where the similarity with the LHC program ends: there is no international consensus to pursue only one experiment and already on-going experiments, notably NOvA in the U.S. and $\mathrm{T} 2 \mathrm{~K}$ in Japan, will be making inroads into the very question of leptonic CP violation. While it seems very unlikely that the on-going experiments will achieve a $5 \sigma$ discovery, a $3 \sigma$ evidence for leptonic $\mathrm{CP}$ violation may be conceivably obtained. Thus future long-baseline experiments will not be entering a terra incognita but will be tasked with the exploration and mapping of terrain seen before. Therefore, precision measurements and a comprehensive set of physics goals is the real scientific objective.

\section{Future prospects}

First tentative hints for leptonic CP violation became apparent earlier this year [1] and since more global fits have been performed [2] indicating a preference for a value of the leptonic CP phase around $\delta \simeq-\pi / 2$. This hint for CP violation currently is at $1-2 \sigma$ level and thus may be nothing more than a statistical fluctuation. On the other hand, both T2K and NOvA do consistently report $v_{e}$ appearance rates at the upper end of the possible range, which is what is expected if $\delta=-\pi / 2$.

The T2K collaboration has formulated a plan, including an approved upgrade of the proton accelerator to increase beam power to $1.2 \mathrm{MW}$, which on a timescale of about a decade will allow $\mathrm{T} 2 \mathrm{~K}$ to reach a $3 \sigma$ rejection of leptonic $\mathrm{CP}$ conservation, assuming the current best-fit value is close to the true value of $\delta$ [3]. In combination with continued NOvA running, current NOvA data represent only about $1 / 6$ of the approved number of protons on target, and the precise determination of $\theta_{13}$ by Daya Bay [4] there will a be a good determination of the leptonic CP phase by 2025 . Also, the question whether $\theta_{23}$ is maximal can be effectively addressed by this data in particular when combined with atmospheric neutrino data, which Super-Kamiokande continues to accumulate. Another crucial piece of information is the neutrino mass hierarchy and while current data seems to have no particular preference, future data from NOvA has the potential to answer this question without any ambiguity. Then, of course, there is JUNO and possibly PINGU all trying to address the same question. It appears, therefore, likely that the question of the mass hierarchy will be settled before too long.

A reasonably good proxy for the actual physics reach of a long-baseline experiment is given by the total number of events it accumulates in the $v_{\mu} \rightarrow v_{e}$ appearance channel. The time evolution is shown in Fig. 1, assuming current best fit values: the next decade will see an order of magnitude increase in these numbers and the statistical errors will drop below 5\%. DUNE has to run for about 3 years to double the global event sample in the appearance channel and even at the end of its planned run it only will have roughly tripled the available global data. Also, by the time DUNE data starts to dominate the global data set, this global data set will comprise about 1500 events, with a corresponding statistical accuracy of $2.6 \%$. This excellent statistical accuracy has to be matched by a corresponding and ideally somewhat smaller systematic uncertainty - otherwise the massive investment in these experiments is wasted. 


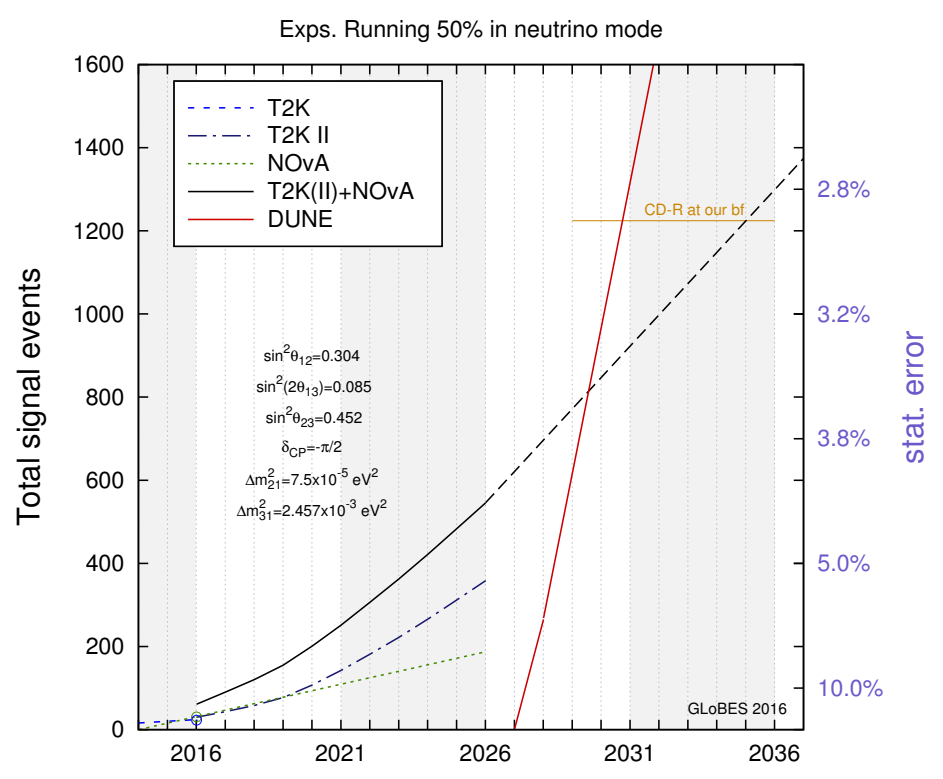

Figure 1: Shown is the time evolution of the total $v_{\mu} \rightarrow v_{e}$ appearance rate for the experiments named in the legend. The assumption is that $\delta=-\pi / 2$ and the mass hierarchy is normal. The axis label on the right-hand vertical axis corresponds to $1 / \sqrt{N}$, with $N$ being the number of events shown on the left-hand vertical axis and thus, represents the statistical accuracy of the data set. The DUNE run plan is based on [5, 6, 7]. The NOvA run plan is based on [8]. The T2K run plan is based on [3].

\section{Road to precision}

There are two challenges for a precision long-baseline experiment: determining the appearance rate and determining the neutrino energy. The rate uncertainty is driven by the knowledge of the beam flux and neutrino interaction cross section. Using a pion-decay, horn-focused beam, the flux knowledge is at best at the $10 \%$ level despite many years of efforts to improve the state of the art by hadron production measurements. In realization of this limitation all long-baseline experiments employ a near detector or a suite of near detectors. Following the argument of Ref. [9], the problem lies in the fact that the near detector, at best, measures the product of beam flux and cross section and thus to know either one, the other quantity is required; there are fewer observables than unknowns. In reactor experiments like Daya Bay the problem is solved by measuring the product of the identical cross section, in this case for inverse beta decay, and the beam flux, in this case $\bar{v}_{e}$, in the near and far detectors, which have the same physical dimensions and characteristics within a fraction of a percent. In inverse beta decay a unique and clean flavor tag is obtained and there is no doubt about the underlying micro-physics, since neutron decay is very well understood. The source is a point source and thus the geometrical acceptance difference between near and far detectors is given by the square of the ratio of baselines, which in turn can be measured with centimeter precision. Thus, the oscillation probability can be extracted by simply taking the ratio of far to near detector data corrected for geometric acceptance.

In a long-baseline experiments none of the conditions which allowed Daya Bay to succeed is met: cross sections in near and far detectors are different since the neutrino flavor and energy 
distribution is different. The beam flux and flavor composition seen by the near detector is different even in absence of oscillation because of the complicated acceptance. The near and far detector are not the same size, do not use the same technology and sometimes not even the same target material. The near detector sees the decay pipe as line source whereas the far detector sees it as point source. Relating the observable quantities to the underlying micro-physics in an event requires a precise understanding of the micro-physics, which we lack. Instead, we rely on Monte Carlo simulations, which have been tuned to existing data, for event identification. The limitations of this approach are exemplified by the fact that basically no two cross section measurements performed in a neutrino beam ever seem to to agree with each other.

Finally, the need to reconstruct the neutrino energy precisely is a design feature of experiments using a wide-band neutrino beam and all the physics benefits of covering a range of $L / E$-values rely on this reconstruction. Again, the lack of an understanding of the micro-physics of neutrinonucleus interactions prevents accurate neutrino energy reconstruction because the observable signatures do not have an understood relation to neutrino energy. It has been shown that approximate schemes like the exploitation of quasi-elastic kinematics do not provide sufficient accuracy for the next generation of experiments [10,11] and also calorimetric methods have their limitations [12], in particular with respect to neutral secondary particles like neutrons. In particular, for the determination of $\mathrm{CP}$ violation this is a major issue [13].

Therefore, even an ideal near detector seems to be insufficient to resolve the systematics problem in long-baseline experiments since it ultimately provides fewer observables than unknowns. The hope that the multitude of different event types, charged current quasi elastics, charged current single pion etc. will provide a sufficient number of observables is naive: they only can constrain each other if we connect them with a micro-physical model, which we do not have. It is a fallacy to confuse existing Monte Carlo event generators with an actual understanding of the micro-physics as borne out by the great difficulty to reconcile any new measurement of exclusive neutrino cross sections with existing ones, to quote from the most recent MINERvA publication [14]: "Unlike the measurements of the individual processes (quasi- elastic, pion production) the total cross section measurements agree with the GENIE simulation and prior data to within their uncertainties [...]" (emphasis added). Given that the MINERvA experiments in many ways represents the state of the art in neutrino scattering, this is discouraging, despite the success for inclusive cross sections: neutrino energy reconstruction desperately relies on an understanding of the exclusive interaction channels.

Usually when the situation seems hopeless on the experimental side, we turn to theory to provide the needed answers. It is obvious that describing bound state multi-nucleon systems in the ground state is a daunting task and so far can be only achieved by using phenomenological Hamiltonians and for nuclei lighter than $A=12$. The problem at hand is however not to know the ground state (or the low-lying excitations) but the response to energy transfers well into the $\mathrm{GeV}$-range, which requires a relativistic treatment. Once the hard scattering event has taken place we also need to understand how the reaction products get out of the nucleus, a problem typically summarized under the term final-state interactions. Recently, Benhar [15] points out that many different calculational approaches based on very different assumption seem to yield the same result, which is puzzling. The role of electron scattering data can not be overstated, since we can exploit fully exclusive kinematics to separate the various micro-physical contributions and any model of 
neutrino interactions must reproduce electron scattering data. Fortunately, a program is underway to obtain this crucial data for argon [16].

\section{Summary}

We are very fortunate in neutrino physics having found neutrino oscillation, and by association that neutrinos have a mass; also, we find large mixing angles, including a quite sizable value of $\theta_{13}$. Thus, the stage is set to study genuine three-flavor effects and to, hopefully, find leptonic $\mathrm{CP}$ violation. There is a vibrant ongoing experimental effort, spearheaded by NOvA and T2K and we have won approval for DUNE. The future of neutrino physics lies in precision studies of longbaseline neutrino oscillation and while a large $\theta_{13}$ allows to accumulate significant event samples we also will need concomitant control of systematical uncertainties.

The choice of pion-decay, horn-focused neutrino beams implies poor knowledge of the primary neutrino flux, which combined with our lack of understanding of neutrino-nucleus interactions presents a challenge. In this note we reiterate the argument previously made in the literature that even a capable near detector complex can not meet this challenge. The state of theory is such that it is at best unclear whether theory can provide the missing answers. Let us assume, that there will a be a breakthrough in theory providing a full model of neutrino-nucleus interaction including final state interactions. This model will not be based on the Standard Model Lagrangian or any other first-principles calculations; given the complexity of the problem it has to be based on phenomenological insights and appropriate approximations. Thus, even if we all agree that we have the "right" model, we will need to test this model at the level of accuracy we intend to use it at. Therefore, the neutrino community needs to seriously think about an experimental neutrino scattering program to accompany the long-baseline oscillation program, see for instance $[17,18]$.

\section{Acknowledgments}

This work was supported by the U.S. Department of Energy Office of Science under award number DE-SC0009973.

\section{References}

[1] A. Marrone, "Status and prospects of global analyses of neutrino mass-mixing parameters." Talk at Neutrino 2016, Jul, 2016.

[2] I. Esteban, M. C. Gonzalez-Garcia, M. Maltoni, I. Martinez-Soler and T. Schwetz, Updated fit to three neutrino mixing: exploring the accelerator-reactor complementarity, 1611.01514.

[3] K. Abe et al., Proposal for an Extended Run of T2K to $20 \times 10^{21}$ POT, 1609.04111.

[4] DAYA BAY collaboration, F. P. An et al., Measurement of electron antineutrino oscillation based on 1230 days of operation of the Daya Bay experiment, 1610.04802.

[5] DUNE collaboration, R. Acciarri et al., Long-Baseline Neutrino Facility (LBNF) and Deep Underground Neutrino Experiment (DUNE), 1512.06148.

[6] DUNE collaboration, T. Alion et al., Experiment Simulation Configurations Used in DUNE CDR, 1606.09550 . 
[7] C. J. Mossey, "News from LBNF.” Talk at DUNE collaboration meeting, May, 2016.

[8] P. Vahle, "New results from NOvA.” Talk at Neutrino 2016, Jul, 2016.

[9] P. Huber, M. Mezzetto and T. Schwetz, On the impact of systematical uncertainties for the CP violation measurement in superbeam experiments, JHEP 03 (2008) 021, [0711.2950].

[10] P. Coloma, P. Huber, C.-M. Jen and C. Mariani, Neutrino-nucleus interaction models and their impact on oscillation analyses, Phys. Rev. D89 (2014) 073015, [1311.4506].

[11] A. M. Ankowski, O. Benhar, P. Coloma, P. Huber, C.-M. Jen, C. Mariani et al., Comparison of the calorimetric and kinematic methods of neutrino energy reconstruction in disappearance experiments, Phys. Rev. D92 (2015) 073014, [1507.08560].

[12] A. M. Ankowski, P. Coloma, P. Huber, C. Mariani and E. Vagnoni, Missing energy and the measurement of the CP-violating phase in neutrino oscillations, Phys. Rev. D92 (2015) 091301, [1507.08561].

[13] P. Coloma and P. Huber, Impact of nuclear effects on the extraction of neutrino oscillation parameters, Phys. Rev. Lett. 111 (2013) 221802, [1307.1243].

[14] MINERvA collaboration, J. Devan et al., Measurements of the Inclusive Neutrino and Antineutrino Charged Current Cross Sections in MINERvA Using the Low-v Flux Method, Submitted to: Phys. Rev. D (2016), [1610.04746].

[15] O. Benhar, Interpretation of the Neutrino-Nucleus Cross Section, 1612.01473.

[16] O. Benhar et al., Measurement of the Spectral Function of ${ }^{40}$ Ar through the $\left(e, e^{\prime} p\right)$ reaction, 1406.4080 .

[17] NUSTORM collaboration, D. Adey et al., nuSTORM - Neutrinos from STORed Muons: Proposal to the Fermilab PAC, 1308.6822.

[18] NUPRISM collaboration, S. Bhadra et al., Letter of Intent to Construct a nuPRISM Detector in the J-PARC Neutrino Beamline, 1412.3086. 